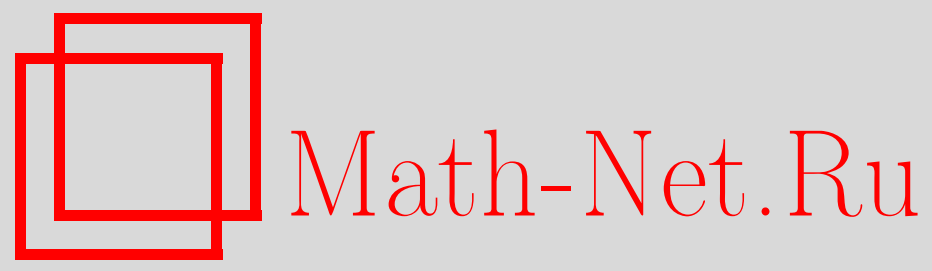

А. В. Ипатова, В. Э. Вильдеман, Построение материальных функций неупругого деформирования алюминиевого сплава Д16Т по результатам испытаний на растяжение и кручение, Вестн. Сам. гос. техн. унта. Сер. Физ.-мат. науки, 2012, выпуск 4(), 106-114

DOI: https://doi.org/10.14498/vsgtu1106

Использование Общероссийского математического портала Math-Net.Ru подразумевает, что вы прочитали и согласны с пользовательским соглашением

http://www.mathnet.ru/rus/agreement

Параметры загрузки:

IP: 54.162 .127 .20

26 апреля 2023 г., 15:34:15

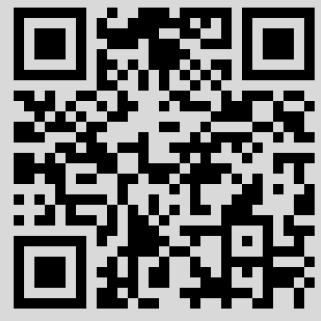




\title{
ПОСТРОЕНИЕ МАТЕРИАЛЬНЫХ ФУНКЦИЙ НЕУПРУГОГО ДЕФОРМИРОВАНИЯ АЛЮМИНИЕВОГО СПЛАВА Д16Т ПО РЕЗУЛЬТАТАМ ИСПЫТАНИЙ НА РАСТЯЖЕНИЕ И КРУЧЕНИЕ
}

\author{
А.В. Ипатова, В.Э. Вилъдеман \\ Пермский национальный исследовательский политехнический университет, \\ 614990, Россия, Пермь, пр. Комсомольский, 29а. \\ E-mails: cem_ipatova@mail.ru, wildemann@pstu.ru
}

\begin{abstract}
Рассмотрены тензорные модели накопления повреждений для изотропных материалов. Определенъ материальные функиии неупругого деформирования алюминиевого сплава Д16Т на основе экспериментальных данных, полученных в опытах на растяжение, кручение, растяжение с кручением при различных соотношениях осевых и сдвиговых дебормаций. Предложены аппроксимации опытных данных аналитическими выражениями, включающими в себя зависимость от первого и второго инвариантов тензора деформаций. Сопоставление экспериментальных и теоретических зависимостей свидетельствует об адекватности предложенных математических моделей.
\end{abstract}

Ключевые слова: материальная функиия, неупругое деформирование, результаты испытаний, аппроксимачия, инвариант тензора дебормаций, алюминиевый сплав.

Реализация уточненных расчётов ответственных конструкций требует развития методов экспериментальных исследований [1-4] и математического моделирования неупругого деформирования материалов в условиях сложного напряженного состояния [5-7]. Построение и обоснование феноменологических моделей накопления повреждений, которому посвящено большое число работ, в частности [8-14], не теряет своей актуальности. В ряде работ процесс накопления повреждений осуществляется с использованием скалярного или тензорного (второго или четвертого ранга) параметра повреждённости.

Феноменологическое описание процессов накопления повреждений, приводящих к изменению деформационных характеристик материала, и установление связи тензора напряжений $\sigma$ с тензором деформаций $\varepsilon$ может быть осуществлено с использованием тензора повреждённости четвертого ранга $\Omega$ в виде $[13,16]$

$$
\sigma_{i j}=C_{i j k l}\left(I_{k l m n}-\Omega_{k l m n}\right) \varepsilon_{m n},
$$

где $C$ - тензор упругих модулей, $I_{k l m n}=1 / 2\left(\delta_{k m} \delta_{l n}+\delta_{k n} \delta_{l m}\right)$ - компоненты единичного тензора, $\delta_{k n}-$ символ Кронекера.

Тензор-оператор повреждённости $\Omega$ однозначно определяется процессом деформирования или нагружения. Зависимость свойств материала от уровня деформаций или напряжений, параметров циклического нагружения при усталости материалов, времени (в частности, при описании процессов ползучести и релаксации), температуры или других факторов также может быть учтена с помощью тензора повреждённости.

Анастасия Владимировна Ипатова, инженер, Центр экспериментальной механики. Валерий Эрвинович Вилъдеман (д.ф.-м.н., проф.), профессор, каф. механики композиционных материалов и конструкций. 
Ограничившись рассмотрением изотропных материалов, процесс неупругого деформирования можно описать с помощью изотропного тензора повреждённости

$$
\Omega_{k l m n}=\omega_{1} \delta_{k l} \delta_{m n}+\omega_{2}\left(\delta_{k m} \delta_{l n}+\delta_{k n} \delta_{l m}\right) .
$$

Соотношения (1) в этом случае сводятся к следующим [16]:

$$
\begin{gathered}
\sigma_{i j}=\left[3 K(1-\kappa) V_{i j m n}+2 G(1-g) H_{i j m n}\right] \varepsilon_{m n}, \\
V_{i j k l}=\delta_{i j} \delta_{k l} / 3, \quad H_{i j k l}=I_{i j k l}-V_{i j k l}, \quad \kappa=3 \omega_{1}+2 \omega_{2}, \quad g=2 \omega_{2},
\end{gathered}
$$

где $K$ и $G$ - упругие модули объемного сжатия и сдвига. Величины $\kappa$ и $g$ выражают изменение деформационных свойств, определяющих поведение материалов при гидростатическом давлении и чистом сдвиге соответственно.

Из соотношений (2) следует, что инварианты тензора напряжений

$$
j_{\sigma}^{(1)}=\sigma_{k k} / 3, \quad j_{\sigma}^{(2)}=\sqrt{\breve{\sigma}_{i j} \breve{\sigma}_{i j}}
$$

связаны с инвариантами тензора деформаций

$$
j_{\varepsilon}^{(1)}=\varepsilon_{k k}, \quad j_{\varepsilon}^{(2)}=\sqrt{\breve{\varepsilon}_{i j} \breve{\varepsilon}_{i j}},
$$

при записи которых использованы обозначения

$$
\breve{\sigma}_{i j} \equiv \sigma_{i j}-\sigma_{k k} \delta_{i j} / 3, \quad \breve{\varepsilon}_{i j} \equiv \varepsilon_{i j}-\varepsilon_{k k} \delta_{i j} / 3,
$$

уравнениями

$$
j_{\sigma}^{(1)}=K(1-\kappa) j_{\varepsilon}^{(1)}, \quad j_{\sigma}^{(2)}=2 G(1-g) j_{\varepsilon}^{(2)},
$$

где $\kappa=\kappa\left(j_{\varepsilon}^{(1)}, j_{\varepsilon}^{(2)}\right), g=g\left(j_{\varepsilon}^{(1)}, j_{\varepsilon}^{(2)}\right)$.

В общем случае сложного напряжённо-деформированного состояния инварианты тензора напряжений связаны с инвариантами тензора деформаций соотношениями, определяющими материальные функции неупругого деформирования $j_{\sigma}^{(1)}=f\left(j_{\varepsilon}^{(1)}, j_{\varepsilon}^{(2)}\right), j_{\sigma}^{(2)}=\varphi\left(j_{\varepsilon}^{(1)}, j_{\varepsilon}^{(2)}\right)$.

Особенность определяющих соотношений (2) заключается в том, что в отличие от теории малых упругопластических деформаций они позволяют учесть нелинейную зависимость относительного изменения объема от гидростатического давления, что может иметь существенное значение для описания поведения пористых материалов и композитов.

Поведение материала при одноосном растяжении описывается уравнениями

$$
\begin{gathered}
\sigma_{33}=E(1-e) \varepsilon_{33}, \quad \varepsilon_{11}=\varepsilon_{22}=-\nu(1-\eta) \varepsilon_{33}, \\
e=\frac{G \kappa(1-g)+3 K g(1-\kappa)}{G(1-g)+3 K(1-\kappa)}, \quad \eta=\frac{K G(\kappa-g)}{(K-2 G / 3)[K(1-\kappa)+2 G / 3(1-g)]},
\end{gathered}
$$

где $E$ и $\nu$ - соответственно модуль Юнга и коэффициент Пуассона материала в неповреждённом состоянии.

Модель деформирования, построенная на основе скалярной функции повреждённости $\left(\Omega_{k l m n}=\Omega I_{k l m n}\right)$, описывает накопление повреждений, при котором относительное изменение всех деформационных свойств происходит 
одинаково. В рамках этой модели для изотропного материала предполагается, что $\kappa=g, \nu=$ const. Скалярная функция $\Omega$ эквивалентна параметру повреждённости Качанова-Работнова.

Если компоненты тензора $\Omega$ изотропного материала определить таким образом, чтобы $\kappa=0$, то есть не учитывать неупругое изменение объёма, то функция повреждённости $g$ совпадёт с функцией пластичности А. А. Ильюшина, а соотношения (2) - с уравнениями теории малых упругопластических деформаций при активном нагружении.

Построение введённых материальных функций неупругого деформирования в более общих случаях требует отработки методик и получения результатов экспериментального исследования поведения материалов в условиях сложного напряжённого состояния.

Применимость моделей накопления повреждений может быть оценена, в частности, на основе экспериментальных данных, полученных в опытах на растяжение, кручение, а также пропорциональное растяжение с кручением.

Как известно, функции (или константы), по которым можно полностью восстановить связь тензора напряжений и тензора деформаций, следуя определяющим соотношениям, описывающим данную модель механики деформируемого твёрдого тела, называются материальными функциями (или константами). Конкретный вид материальных функций, входящих в определяющие соотношения, устанавливается на основе анализа экспериментальных данных. В качестве аргументов используются инварианты тензоров деформаций и напряжений, определяемые согласно соотношениям (3)-(5) по формулам

$$
\begin{gathered}
j_{\varepsilon}^{(1)}=\frac{1}{3}\left(\sigma_{11}+\sigma_{22}+\sigma_{33}\right), \\
j_{\sigma}^{(2)}=\frac{1}{\sqrt{3}} \sqrt{\left(\left(\sigma_{11}-\sigma_{22}\right)^{2}+\left(\sigma_{11}-\sigma_{33}\right)^{2}+\left(\sigma_{22}-\sigma_{33}\right)^{2}+6\left(\tau_{12}^{2}+\tau_{13}^{2}+\tau_{23}^{2}\right)\right)}, \\
j_{\varepsilon}^{(1)}=\varepsilon_{11}+\varepsilon_{22}+\varepsilon_{33}, \\
j_{\varepsilon}^{(2)}=\frac{1}{\sqrt{3}} \sqrt{\left(\left(\varepsilon_{11}-\varepsilon_{22}\right)^{2}+\left(\varepsilon_{11}-\varepsilon_{33}\right)^{2}+\left(\varepsilon_{22}-\varepsilon_{33}\right)^{2}+6\left(\varepsilon_{12}^{2}+\varepsilon_{13}^{2}+\varepsilon_{23}^{2}\right)\right)} .
\end{gathered}
$$

Например, поведение упругопластического материала с линейным упрочнением описывается функцией $g=g\left(j_{\varepsilon}^{(1)}, j_{\varepsilon}^{(2)}\right)$ следующего вида:

$$
g= \begin{cases}0, & j_{\varepsilon}^{(2)} \leqslant j_{\varepsilon \text { упр }}^{(2)} \\ \left(1-G^{\prime} / G\right)\left(1-j_{\left.\varepsilon \text { упр } / j_{\varepsilon}^{(2)}\right),}^{(2)}\right. & j_{\varepsilon}^{(2)}>j_{\varepsilon \text { упр }}^{(2)}\end{cases}
$$

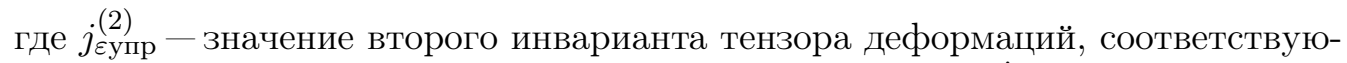
щего пределу упругости рассматриваемого материала; $G^{\prime}$ - модуль упрочнения.

Рассмотрим более подробно экспериментальные данные, полученные на основе опытов, проведённых в Центре экспериментальной механики Пермского национального исследовательского политехнического университета с использованием системы двухосного нагружения Instron 8850, основные принципы работы которой, возможности и технические характеристики изложены в $[3,18,19]$. 
На рис. 1 и 2 представлены экспериментальные диаграммы пропорционального деформирования алюминиевого сплава Д16Т, полученные с использованием тонкостенных трубчатых образцов. Скорость осевого деформирования - 3,33\% в минуту, скорость изменения угла сдвига - 0,052 рад/мин. Удлинения и углы закручивания в рабочих зонах образцов регистрировались с использованием двухосевого экстензометра. Приведённые данные могут быть применены для установления зависимостей первых и вторых инвариантов тензоров напряжений от инвариантов тензоров деформаций.

Для точного определения первого инварианта тензора деформаций при проведении испытаний следует регистрировать поперечные деформации. При использовании полых цилиндрических образцов это связано с техническими трудностями. В связи с этим было принято допущение, что коэффициент Пуассона, определённый по значениям упругих констант и равный 0,235, не изменяется в процессе пластического деформирования. Полученные зависимости инвариантов представлены на рис. 2.

Полученные данные позволяют расчётным путем установить зависимости значений функций повреждённости от значений инвариантов тензоров деформаций. На рис. 3 представлены результаты построения материальной функции $e=e\left(j_{\varepsilon}^{(1)}, j_{\varepsilon}^{(2)}\right)$ по результатам испытаний на одноосное растяжение, растяжение с кручением.

На основании приведённых данных можно сделать вывод о существенной зависимости функции повреждённости от первого инварианта тензора деформаций.
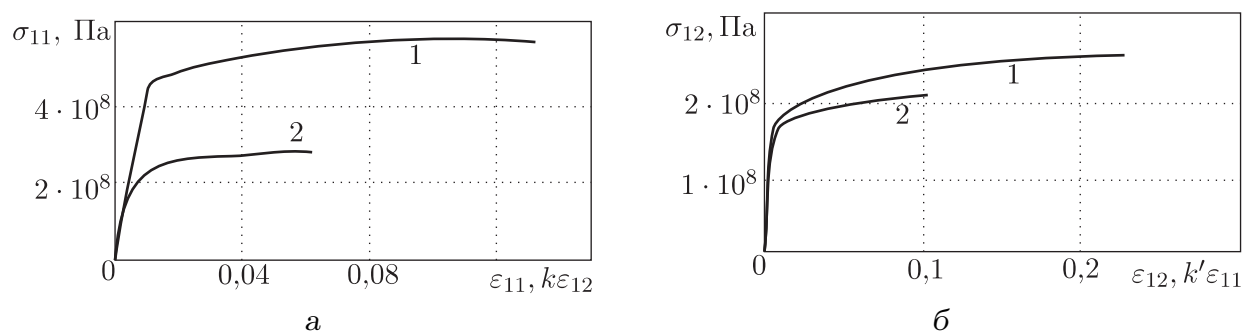

Рис. 1. Экспериментальные диаграммы деформирования: а) 1 - растяжение, 2 - растяжение с кручением при $\left.\varepsilon_{11}=k \varepsilon_{12}, k=0,63 ; \sigma\right) 1-$ кручение, $2-$ растяжение с кручением при $\varepsilon_{12}=k^{\prime} \varepsilon_{11}$, $k^{\prime}=1 / k=1,59$

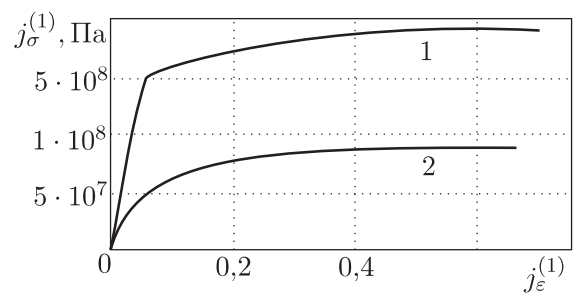

a

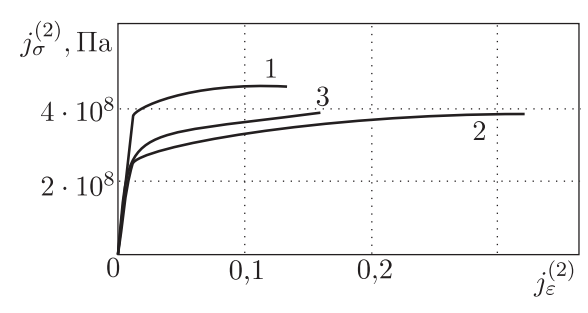

б

Рис. 2. Зависимости инвариантов тензора напряжений от инвариантов тензора деформаций: а) 1 -одноосное растяжение, 2 - растяжение с кручением при $\left.\varepsilon_{11}=k \varepsilon_{12}, k=0,63 ; \sigma\right) 1-$ одноосное растяжение, 2 - кручение, 3 - растяжение с кручением при $\varepsilon_{11}=k \varepsilon_{12}, k=0,63$ 


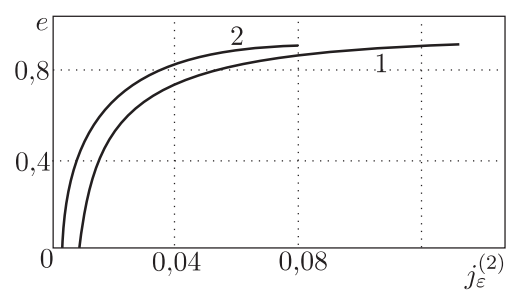

Рис. 3. Построение материальной функции $e=e\left(j_{\varepsilon}^{(2)}\right): 1-$ по результатам испытаний на одноосное растяжение, 2 - растяжение с кручением при $\varepsilon_{11}=k \varepsilon_{12}(k=0,63)$

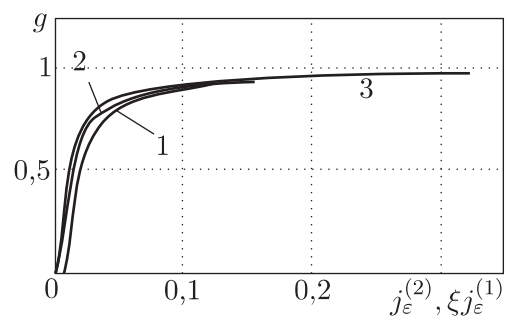

Рис. 4. Построение материальной функции $g=g\left(j_{\varepsilon}^{(1)}, j_{\varepsilon}^{(2)}\right): 1-$ по результатам испытаний на кручение, 2 - растяжение, 3 растяжение с кручением при $\varepsilon_{11}=k \varepsilon_{12}(k=$ $=0,63, \xi=4,643)$

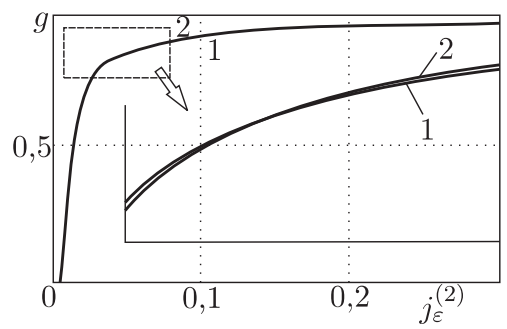

Рис. 5. Построение материальных функций $g=g\left(j_{\varepsilon}^{(2)}\right)$ неупругого деформирования для опыта на кручение: 1 - экспериментальная кривая, $2-$ функция $g=g\left(j_{\varepsilon}^{(2)}\right)$, построенная по формуле (6)
В этом случае коэффициент связи первого и второго инвариантов деформаций, определяемый по формуле $\xi=$ $=j_{\varepsilon}^{(2)} / j_{\varepsilon}^{(1)}$, для случая чистого сдвига стремится к бесконечности, для одноосного растяжения равен 1,903, для пропорционального деформирования при соотношении $\varepsilon_{11}=k \varepsilon_{12}$ и $k=0,63$ составляет 4,643.

На рис. 4 представлена функция повреждённости $g=g\left(j_{\varepsilon}^{(1)}, j_{\varepsilon}^{(2)}\right)$, построенная по результатам механических испытаний на кручение $(\xi=\infty)$, растяжение $(\xi=1,903)$, растяжение с кручением $(k=0,63, \xi=4,643)$.

Рассмотрим вопрос аппроксимации полученных экспериментальных зависимостей и конкретизации моделей неупругого деформирования. Зависимость $g=g\left(j_{\varepsilon}^{(2)}\right)$, полученная на основании опыта на кручение (кривая 1 на рис. 4), без учёта зависимости от первого инварианта может быть аппроксимирована выражением

$$
g= \begin{cases}0, & j_{\varepsilon}^{(2)} \leqslant j_{\varepsilon \text { упр }}^{(2)} \\ A\left(1-j_{\varepsilon \text { упр }}^{(2)} j_{\varepsilon}^{(2)}\right)^{\alpha}, & j_{\varepsilon}^{(2)}>j_{\varepsilon \text { упр }}^{(2)},\end{cases}
$$

где $A, \alpha$ - константы материала.

На основании опытных данных получены значения констант материала для рассматриваемого алюминиевого сплава: $A=0,99 ; \alpha=1,3$. Результаты построения экспериментальной и теоретической кривой представлены на рис. 5 .

На рис. 6, а представлены графические зависимости для опыта на одноосное растяжение. Как видим, теоретическая кривая 2, построенная на основе выражения (6), не достаточно точно описывает полученные экспериментальные данные (кривая 1). Для уточнённого описания опытных значений, полученных при растяжении, предложим следующую модель, учитывающую зависимость материальной функции не только от второго, но и от первого инварианта тензора деформаций:

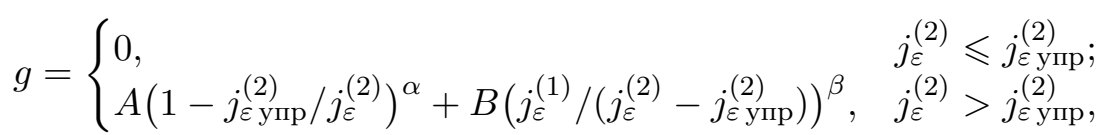

где $B=-0,5 ; \beta=3,57$. 
Данная модель достаточно точно описывает и случай пропорционального нагружения в условиях растяжения с кручением, что отражено на рис. 6, б.

Проведем сравнение теоретических кривых, построенных по формулам (6) (без учета влияния первого инварианта), (7) (с учетом влияния первого инварианта), и экспериментальных кривых, полученных в опыте на одноосное растяжение (кривая 2 на рис. 4) и растяжение с кручением (кривая 3 на рис. 4). Сопоставление результатов отражено на рис. 6.

Как видно, усложненная модель (7) более точно соответствует экспериментальным данным.

С целью получения данных для верификации модели были получены результаты независимых испытаний в условиях сложного напряженно-деформированного состояния при других соотношениях величин осевых и сдвиговых деформаций $(k=0,45 ; k=0,32)$. Результаты построения функций $g=g\left(j_{\varepsilon}^{(1)}, j_{\varepsilon}^{(2)}\right)$ на основе экспериментальных данных приведены на рис. 7. Применим модель (7) для описания полученных данных.

Как показано на рис. 8, обнаруживается хорошее соответствие расчётных и экспериментальных данных.

Таким образом, на основе полученных экспериментальных данных по одноосному растяжению, кручению, растяжению с кручением при различных соотношениях осевых и сдвиговых деформа-

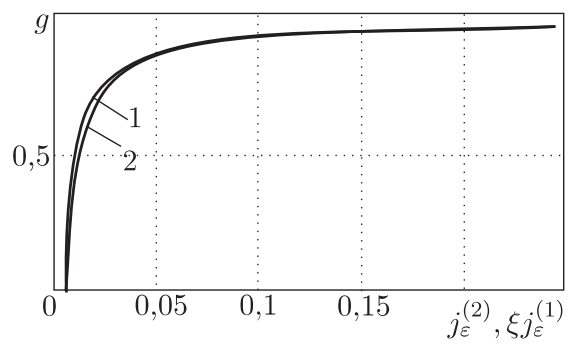

a

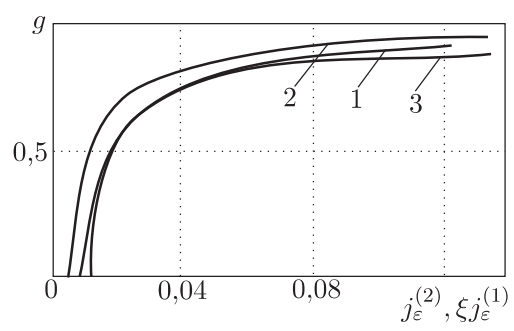

a

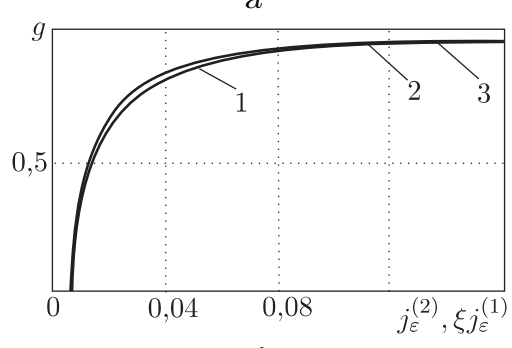

б

Рис. 6. Построение материальных функций $g=g\left(j_{\varepsilon}^{(1)}, j_{\varepsilon}^{(2)}\right)$ неупругого деформирования для опытов на растяжение (а) и растяжение с кручением (б): 1 -экспериментальная кривая, 2 - функция $g=$ $=g\left(j_{\varepsilon}^{(2)}\right)$ без учёта влияния $j_{\varepsilon}^{(1)}, 3$ - функция $g=g\left(j_{\varepsilon}^{(1)}, j_{\varepsilon}^{(2)}\right)$ по формуле (7)

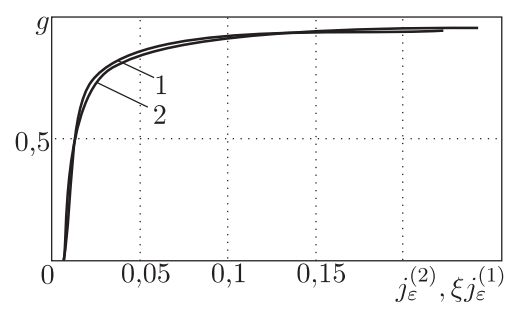

Рис. 7. Построение материальных функций неупругого деформирования на основании опытных данных (растяжение с кручением при различных соотношениях осевых и сдвиговых деформаций $\varepsilon_{11}=$ $\left.=k \varepsilon_{12}\right): 1-$ при $k=0,45(\xi=6,227) ; 2-$ при $k=0,32(\xi=8,553)$

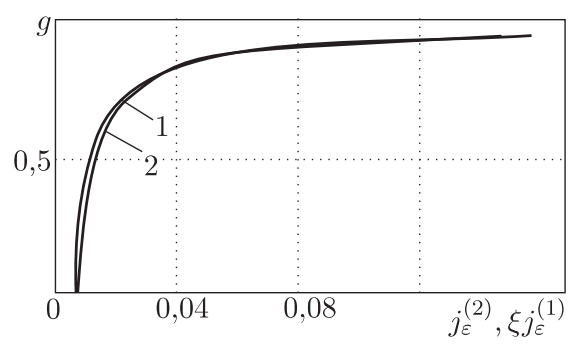

б

Рис. 8. Построение материальных функций неупругого деформирования на основании опытных данных на растяжение с кручением при $\varepsilon_{11}=k \varepsilon_{12}$ : а) $\left.k=0,45, \xi=6,227 ; \sigma\right) k=$ $=0,32, \xi=8,553 ; 1$ - экспериментальные данные, 2 - кривая, построенная по формуле (7) 
ций для алюминиевого сплава построены материальные функции неупругого деформирования, аргументами которых являются, в общем случае, два инварианта тензора деформаций. Предложены аппроксимации опытных данных аналитическими выражениями для материальных функций неупругого деформирования. Сопоставление экспериментальных и теоретических зависимостей свидетельствует об адекватности предложенных математических моделей.

Работа выполнена в рамках государственного контракта № 13.G25.31.0093 от 25 октября 2010 г., а также РФФИ в рамках научного проекта № 12-08-31336 мол_а.

\section{БИБЛИОГРАФИЧЕСКИЙ СПИСОК}

1. Анин Б. Д., Жигалкин В. М. Поведение материалов в условиях сложного нагружения. Новосибирск: CO PAH, 1999. 342 с. [Annin B. D., Zhigalkin V. M. Behavior of Materials under Complex Loading: SO RAN, 1999. 342 pp.]

2. Лебедев А.А., Ковальчук Б. И., Гигиняк Ф. Ф., Ламашевский В. П. Механические свойства конструкционных материалов при сложном напряженном состоянии. Киев: Ин Юpe, 2003. 540 c. [Lebedev A. A., Kovalchuk B.I., Giginyak F. F., Lamashevskii V.P. Mechanical Properties of Structural Materials with a Complex Stress State. Kiev: In Yure, 2003. 540 pp.]

3. Вильдеман В. Э., Третъяков М. П., Третъякова Т. В., Булъбович Р. В., Словиков С. В., Бабушкин А.В., Ильиных А.В., Лобанов Д. С., Ипатова А.В. Экспериментальные исследования свойств материалов при сложных термомеханических воздействиях / ред. В.Э. Вильдеман. М.: Физматлит, 2012. 204 с. [Vil'deman V. E., Tret'yakov M. P., Tret'yakova T.V., Bul'bovich R.V., Slovikov S.V., Babushkin A.V., Il'inykh A.V., Lobanov D.S., Ipatova A. V. Experimental investigations of materials properties at combined thermo-mechanics influences / ed. V. E. Vil'deman. Moscow: Fizmatlit, 2012. 204 pp.]

4. Вассерман Н. Н., Вилъдеман В. Э., Крюков А. А., Третъяков М. П. Исследование зако-

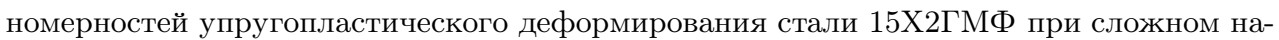
пряженном состоянии // Вестн. Перм. госуд. техн. ун-та. Механика, 2010. № 2. С. 3447. [Vasserman N. N., Vil'demann V. E., Kryukov A.A., Tret'yakov M. P. Investigation of the elastoplastic deformation laws of steel 15H2GMF on a complex stress state // Perm State Technical University Mechanics Bulletin, 2010. no. 2. Pp. 34-47].

5. Вилъдеман B.Э., Ипатова А.В. Численное исследование полей деформаций и напряжений однонаправлено-армированных волокнистых композиционных материалов стохастической структуры // Механика композиционных материалов и конструкиий, 2010. Т. 16, № 3. С. 421-435; англ. пер.: Numerical investigation of strain and stress fields in unidirectionally reinforced fibrous composite materials of stochastic structure // International Journal of Nanomechanics Science and Technology, 2010. Vol.1, no. 4. Pp. 273-290.

6. Вилъдеман В.Э., Илъиных А.В. Моделирование процессов структурного разрушения и масштабных эффектов разупрочнения на закритической стадии деформирования неоднородных сред// Физическая мезомеханика, 2007. Т. 10, № 4. С. 23-29. [Vil'demann V.E., Il'inykh A.V. Simulation of structural failure and scale effects of softening at the post-critical deformation stage in heterogeneous media// Fizicheskaya Mezomekhanika, 2007. Vol. 10, no. 4. Pp. 23-29].

7. Ильиных А.В., Вилъдеман В.Э. Закономерности механического поведения зернистых композитов, связанные с формой и размерами элементов структуры // Becmн. Перм. нач. исслед. политехн. ун-та. Механика, 2011. №4. C. 49-61. [Il'inykh A. V., Vil'demann V. E. Principles of mechanical behavior of granular composites, connected with form and sizes of elements of structure // PNRPU Mechanics Bulletin, 2011. no. 4. Pp. 49$61]$. 
8. Качанов Л. М. О времени разрушения в условиях ползучести // Изв. АН СССР. ОТН, 1958. № 8. C. 26-31; Kachanov L. M. Rupture time under creep conditions // Int. J. Fracture, 1999. Vol. 97, no. 1-4. Pp. 11-18.

9. Работнов Ю.Н. О механизме длительного разрушения/ В сб.: Вопросы прочности материалов и конструкиий. М.: АН CCCP, 1959. C. 5-7. [Rabotnov Yu. N., A mechanism of the long term fracture/ In: Voprosy prochnosti materialov i konstruktsii. Moscow: AN SSSR, 1959. Pp. 5-7].

10. Тамуж В.П., Лагздинъш А.Ж. Вариант построения феноменологической теории разрушения // Механика полимеров, 1968. Т. 4, №4. С. 638-647; Tamuzh V. P., Lagzdyn'sh A. Zh. A variant of the phenomenological theory of fracture// Polymer Mechanics, 1968. Vol. 4, no. 4-6. Pp. 493-500.

11. Ильюшин А.А. Победря Б. Е. Основы математической теории термовязкоупругости. M.: Наука, 1970. 280 c. [Il'yushin A. A., Pobedrya B. E. Foundations of the mathematical theory of thermal viscoelasticity. Moscow: Nauka, 1970. 280 pp.]

12. Novozhilov $V . V$. Perspective in phenomenological approach to the problem of fracture/ В сб.: Mechanics of Deformable Bodies and Structures. Moscow: Mashonosroenie, 1975. 349-359 c.

13. Соколкин Ю. В., Ташкинов А. А. Механика деформирования и разрушения структурно-неоднородных тел. М.: Наука, 1984. 115 с. [Sokolkin Yu. V., Tashkinov A. A. Mechanics of Deformation and Failure of Structural Heterogeneous Bodies. Moscow: Nauka, 1984. 115 pp.]

14. Березин A.B. Влияние повреждений на деформационные и прочностные характеристики твердых тел. М.: Наука, 1990. 135 с. [Berezin A. V. A influence of damage on the deformation and strength characteristics of the solid media. Moscow: Nauka, 1990. 135 pp.]

15. Радченко В. П. Математическая модель неупругого деформирования и разрушения металлов при ползучести энергетического типа // Вестн. Сам. гос. техн. ун-та. Сер. Физ.-мат. науки, 1996. № 4. C. 43-63. [Radchenko V.P. The mathematical model of inelastic deformation and failure of the metals by energy-type creep// Vestn. Samar. Gos. Tekhn. Univ. Ser. Fiz.-Mat. Nauki, 1996. no. 4. Pp. 43-63].

16. Вильдеман В. Э., Соколкин Ю. В., Ташкинов А. А. Механика неупругого деформирования и разрушения композиционных материалов / ред. Ю. В. Соколкин. М.: Физматлит, 1997. 288 с. [Vil'deman V.E., Sokolkin Yu. V., Tashkinov A. A. Mechanics of inelastic deformation and fracture of composite materials / ed. Yu. V. Sokolkin. Moscow: Fizmatlit, 1997. 288 pp.]

17. Пантелеев И.А., Плехов О.А., Наймарк О.Б. Некоторые автомодельные закономерности развития поврежденности при квазихрупком разрушении твердых тел// $B b$ числительная механика сплошных сред, 2011. Т. 4, №1. C. 90-100. [Panteleev I. A., Plekhov O.A., Naymark O.B. Self-similarity mechanisms of damage growth in solids experiencing quasi-brittle fracture// Vychislitel'naya Mekhanika Sploshnykh Sred, 2011. Vol. 4, no. 1. Pp. 90-100].

18. Вилъдеман В.Э., Санникова Т. В., Третъяков М. П. Экспериментальное исследование закономерностей деформирования и разрушения материалов при плоском напряженном состоянии // Проблемы машиностроения и надежности машин, 2010. № 5. С. 106111; Vil'deman V.E., Sannikova T.V., Tret'yakov M.P. Experimental investigation of material deformation and failure regularities in a flat stressed state // Journal of Machinery Manufacture and Reliability. Vol. 39, no. 5. Pp. 492-496. 
19. Вильдеман В.Э., Бабушкин А.В., Третъяков М.П., Ильиных А.В., Третъякова Т. В., Ипатова А.В., Словиков С.В., Лобанов Д. С. Механика материалов. Методы и средства экспериментальных исследований: учебное пособие / ред. В. Э. Вильдеман. Пермь: Перм. нац. исслед. политехн. ун-т, 2011. 165 с. [Vil'deman V.E., Babushkin A.V., Tret'yakov M.P., Il'inykh A.V., Tret'yakova T.V., Ipatova A.V., Slovikov S. V., Lobanov D.S. Mechanics of Materials. Methods and means of experimental studies: Tutorial/ ed. V. E. Vil'deman. Perm: Perm National Research Polytechnic University, 2011. 165 pp.]

Поступила в редакцию $07 / \mathrm{VII} / 2012$;

в окончательном варианте - 19/X/2012.

\section{MSC: 74C05}

\section{CONSTRUCTION OF MATERIAL FUNCTIONS OF ALUMINUM ALLOY D16T INELASTIC DEFORMATION BASED ON THE RESULTS OF TESTS OF TENSION AND TORSION}

\section{A. V. Ipatova, V.E. Vil'deman}

Perm State National Research Polytechnical University, 29a, Komsomolskiy prospekt, Perm, Russia, 614990.

E-mails: cem_ipatova@mail.ru, wildemann@pstu.ru

The tensor models of damage accumulation for isotropic materials are considered. The material functions of aluminum alloy D16T inelastic deformation based on the results of tests of tension, torsion, tension with torsion with different relations of axial and shear deformations are defined. Approximations of experimental data by analytical expressions are proposed. These expressions include the dependence on the first and second invariants of the strain tensor. Comparison of experimental and theoretical dependences attests to adequacy of the proposed mathematical models.

Key words: material function, inelastic deformation, test results, approximation, invariant of the strain tensor, aluminum alloy.

Original article submitted 07/VII/2012; revision submitted 19/X/2012.

Anastasiya V. Ipatova, Engineer, Center of Experimental Mechanics.

Valeriy E. Vil'deman (Dr. Sci. (Phys. \& Math.)), Professor, Dept. of Mechanics of Composition Materials \& Structures. 